d'un bêta-bloquant, ou vice-versa. Tout était fait pour éviter une telle association qui aurait gêné dans l'interprétation des résultats;

3. L'attitude vis-à-vis de la surcharge pondérale et des autres facteurs de risque (comme le tabac) n'a pas été codifiée et fut probablement différente d'un médecin à l'autre; 4. Environ $40 \%$ des malades inclus ont interrompu secondairement le traitement initialement prescrit, ce qui représente un pourcentage important. C'est pourquoi l'analyse statistique a été faite à partir de la randomisation initiale dans les différentes catégories de traitement, comme si tous les malades avaient poursuivi jusqu'au terme de l'essai le traitement déterminé par le tirage au sort (ce qui manifestement n'a pas été le cas pour beaucoup d'entre eux). Le même principe d'analyse a été appliqué dans l'étude EWPHE pour les mêmes raisons.

Enfin, pour mieux comprendre le débat concernant le traitement de l'HTA modérée, et les difficultés de tels essais thérapeutiques, il faut méditer la première phrase de la conclusion de l'étude MRC: "L'essai a montré que si 850 malades ayant une HTA modérée reçoivent un traitement médicamenteux antihypertenseur actif pendant un an, on préviendra environ 1 accident vasculaire cérébral. Cela représente un bénéfice "important mais rare (c'est-à-dire, concernant une très faible fraction de la population).

J.-P. G.

\footnotetext{
I. Amery A, et al. Mortality and morbidity results from the European Working Party ou High Blood Pressure in the Elderly Trial. Lancet 1985; i: 1349-54.

2. Medical Research Council Working Party. MRC Trial of Treatment of mild hypertension: principal results. $\mathrm{Br}$ med 7 1985; 29 I: 97-104.
}

\title{
Une empreinte individuelle d'ADN
}

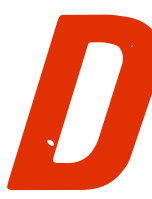

ès que l'on a su analyser des séquences d'ADN, on s'est aperfu que le polymorphisme génétique était beaucoup plus important que ne l'avait fait prévoir l'étude des protéines. Il n'y a certainement pas deux personnes dans le monde qui possèdent la même séquence d'ADN. Toutefois, avec les techniques actuelles, un séquenfage de masse demanderait des siècles. Des chercheurs britanniques $[I, 2]$ viennent de court-circuiter le problème de fafon élégante. Ils se sont appuyés sur l'existence de régions répétitives d'ADN dispersées dans le génome. Les éléments répétitifs dont il existe plusieurs types sont constitués de séquences communes de Io à 15 paires de bases. Le polymorphisme résulte du fait que le nombre de ces éléments differe d'un sujet à un autre, en raison probablement de recombinaisons entre séquences similaires avec échange inégal non équationnel (cf. Médecine/sciences $\mathrm{n}^{\circ} 4$, Lexique p. 215, fig. 3). Il se crée ainsi des zones d'ADN dites hypervariables mais qui, une fois établies, sont stables dans les cellules somatiques et peuvent servir à caractériser les individus.

Il a suffi d'hybrider deux sondes, contenant chacune une unité répétitive, avec de l' $A D N$ total digéré par une ou deux enzymes de restriction (Hinf I associée ou non à Sau $\mathrm{U}_{3} \mathrm{~A}$ ) pour obtenir une image complexe, spé- cifique de chaque individu. D'après les auteurs, il n'existe qu'une chance infime (moins de $5 \times 10^{-19}$ ) que deux personnes non apparentées aient la même "empreinte "d'ADN. A l'intérieur d'une même fratrie, cette chance ne dépasserait pas $10^{-8}$, sauf naturellement chez les jumeaux monozygotes.

Ce qui rend la technique utilisable, c'est la stabilité du polymorphisme ainsi observé car le taux de mutation pour chaque allèle est très faible. C'est ainsi que les jumeaux monozygotes présentent une image identique. Les caractères (taille des bandes) sont transmis selon une hérédité mendélienne; enfin, l'image initiale se maintient dans les cellules qui se divisent en culture.

La méthode permet donc l'identification des individus (par exemple en médecine légale), des lignées cellulaires, ainsi que les recherches de paternité puisque, chez les descendants, la moitié des fragments sont d'origine paternelle et peuvent être identifiés comme tels.

Tout cela avec à peine plus d'un microgramme d'ADN, tiré de quelques gouttes de sang...

J.-C. D.

I. Jeffreys AJ, Wilson V, Thein SL. Hypervariable minisatellite regions in human DNA. Nature 1985; 314:67-73.

2. Jeff reys $A J$, Wilson $V$, Thein SL. Individual specific - fingerprints of human DNA. Nature 1985; $316: 76-9$.

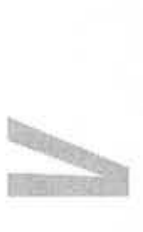

